\title{
Effect of Surfactant Type and Sonication Energy on the Electrical Conductivity Properties of Nanocellulose-CNT Nanocomposite Films
}

\author{
Sanna Siljander ${ }^{1, *}$, Pasi Keinänen ${ }^{1}$ (D), Anna Räty ${ }^{1}$, Karthik Ram Ramakrishnan ${ }^{1}$, \\ Sampo Tuukkanen ${ }^{2}\left(\mathbb{D}\right.$, Vesa Kunnari ${ }^{3}$, Ali Harlin ${ }^{3}$, Jyrki Vuorinen ${ }^{1}$ (D) and Mikko Kanerva ${ }^{1}$ (D) \\ 1 Laboratory of Materials Science, Tampere University of Technology, FI-33720 Tampere, Finland; \\ pasi.keinanen@tut.fi (P.K.); anna.raty@tut.fi (A.R.); karthik.ramakrishnan@tut.fi (K.R.R.); \\ jyrki.vuorinen@tut.fi (J.V.); mikko.kanerva@tut.fi (M.K.) \\ 2 BioMediTech, Tampere University of Technology, FI-33720 Tampere, Finland; sampo.tuukkanen@tut.fi \\ 3 VTT Research Center, FI-02044 Espoo, Finland; vesa.kunnari@vtt.fi (V.K.); ali.harlin@vtt.fi (A.H.) \\ * Correspondence: sanna.siljander@tut.fi; Tel.: +358-50-3-555-777
}

Received: 15 May 2018; Accepted: 15 June 2018; Published: 20 June 2018

\begin{abstract}
We present a detailed study on the influence of sonication energy and surfactant type on the electrical conductivity of nanocellulose-carbon nanotube (NFC-CNT) nanocomposite films. The study was made using a minimum amount of processing steps, chemicals and materials, to optimize the conductivity properties of free-standing flexible nanocomposite films. In general, the NFC-CNT film preparation process is sensitive concerning the dispersing phase of CNTs into a solution with NFC. In our study, we used sonication to carry out the dispersing phase of processing in the presence of surfactant. In the final phase, the films were prepared from the dispersion using centrifugal cast molding. The solid films were analyzed regarding their electrical conductivity using a four-probe measuring technique. We also characterized how conductivity properties were enhanced when surfactant was removed from nanocomposite films; to our knowledge this has not been reported previously. The results of our study indicated that the optimization of the surfactant type clearly affected the formation of freestanding films. The effect of sonication energy was significant in terms of conductivity. Using a relatively low $16 \mathrm{wt}$. \% concentration of multiwall carbon nanotubes we achieved the highest conductivity value of $8.4 \mathrm{~S} / \mathrm{cm}$ for nanocellulose-CNT films ever published in the current literature. This was achieved by optimizing the surfactant type and sonication energy per dry mass. Additionally, to further increase the conductivity, we defined a preparation step to remove the used surfactant from the final nanocomposite structure.
\end{abstract}

Keywords: nanocellulose; carbon nanotubes; nanocomposite; conductivity; surfactant

\section{Introduction}

Conductive composite materials with micrometer and nanoscale fillers, like metallic powders, carbon black, graphite and carbon fibers, are used in many applications, such as antistatic films and electromagnetic interference (EMI) shielding. Electrical conductivity of $0.01 \mathrm{~S} / \mathrm{cm}$ or higher is required for the composite to be considered conductive, while materials with lower conductivity can be used as antistatic and semiconducting materials. One of the drawbacks with most fillers is that the filler content ratio needs to be as high as $50 \mathrm{wt}$. \% to achieve the percolation threshold (i.e., the critical concentration of filler that corresponds to the sharp rise of conductivity). However, this high filler content ratio might lead to a decrease in the resultant composite's mechanical properties [1,2]. Nanomaterials, such as carbon nanotubes (CNTs) and graphene, play a role in the development of future composite materials. 
For example, CNTs and graphene have been used to toughen matrix polymers [3], to adjust barrier properties of nanocomposite films [4], and to form hierarchical reinforcements [5]. It is possible to attain the percolation threshold in the insulating polymer matrix at a low CNT concentration due to their excellent electrical, mechanical and thermal properties.

Individual CNTs are part of a group of the strongest and most conductive nanomaterials known [6]. Additionally, CNTs can carry higher current density than any other known material, with its highest measured value being $109 \mathrm{~A} / \mathrm{cm}^{2}[7,8]$. However, to obtain an ideal conductive network, the carbon nanotubes have to be well separated and homogenous dispersion should be maintained in the final product. Without efficient dispersion, filler aggregates act as defect sites, which leads to lower mechanical performance $[9,10]$. As the most abundant polymer on earth, cellulose is a promising and well-known material that can be used as a matrix in nanocomposites.

Cellulose is environmentally conscious, low-cost, strong, dimension-stable, non-melting, non-toxic and is a non-metal matrix. The interest towards nanoscale cellulose has increased during the past few years because of its inherent properties, including its good mechanical properties, which are better than those of the respective source biomass material [11]. Cellulose-based micro-/nanofibrils (MFC/NFC) can be extracted from various types of plant fibers using mechanical forces, chemical treatments, enzymes or combinations of these. The most typical approach, however, is to apply wood pulp and mechanical methods such as homogenization, microfluidization, microgrinding and cryocrushing. Finally, after fibrillation, the width of NFC is typically between 5 and $20 \mathrm{~nm}$, with a length of several micrometers. Nanocellulose (NFC) has hydroxyl groups in its structure and is therefore associated with high aspect ratio and strong hydrogen bonds formed between nanocellulose fibers [12]. These bonds enhance mechanical properties and enable the formation of free standing films. A combination of CNTs and cellulose I provides a conductive nanocomposite network. CNT-cellulose composites have been reported to be used as supercapacitor electrodes [13,14], electromagnetic interference shielding devices [15], chemical vapor sensors [16], water sensors [17,18], and pressure sensors [19].

There are different manufacturing methods for the fabrication of CNT-cellulose nanocomposites, but all the methods typically include (1) a phase of dispersing CNTs into a solution, and (2) an impregnation phase into the cellulose substrates (e.g., paper, filter paper) [15,16,20-23]. Alternatively, the dispersion can be used as a wet component with bacterial cellulose [24,25], with cellulose I and regenerated cellulose fibers $[13,18,26]$ or in an aerogel form [17]. The processing of nanocellulose in an aqueous medium is the most common way due to its tendency to react with water, and strong affinity to itself and hydroxyl group containing materials [12]. Chen et al. [27] showed that NFCs and CNTs can form a three-dimensional conductive network structure in a gel-film morphology to achieve high electrical conductivity.

The properties of the nanocellulose-CNT composites are affected by the quality of CNT dispersion, amount of structural and oxidative defects in the graphitic structure of the CNTs, the aspect ratio of the CNTs after the disaggregate treatment, the strength of the matrix, and the interactions between the CNTs and the cellulose matrix. [28] The key challenge in numerous industrial applications is to achieve uniform and stable CNT dispersion. The homogenization phase is vital to maximize the excellent mechanical, electrical and thermal properties of the CNTs and the eco-friendly, strong and low-cost nanocellulose matrix. This is particularly important in the case of submicron- or nanometer-sized particles. In these scales, the surface chemistry plays an important role, managing the particle dispersion within the final product [29]. CNT dispersions are challenging because as the surface area of particles increases, the attractive forces between the aggregates [29] and the high aspect ratio enable the entanglement and bundling of CNTs [30]. There are two phenomena that affect CNT dispersions: nanotube morphology and the forces between the tubes. Entanglement of CNTs occurs due to tube morphology, as well as molecular forces, high aspect ratio, and high flexibility. Dispersing these entangled aggregates is difficult without damaging the nanotubes. Both CNT and aggregate size are expected to play a crucial role in the achieved level of electrical conductivity [31].

Two typical dispersion methods for CNTs include high shear mixing and pure sonication $[13,15,16,19-21,24,32]$. Sonication is based on ultrasonic waves that generate microscopic bubbles 
or inertial cavitation, which produces a shearing action. This results in liquid and suspended particles becoming intensely agitated. Another common technique is to use a centrifuge in one of the processing steps to extract the unwanted agglomerates from the supernatant, but this additional phase takes time and effort and affects the concentration of dispersed particles in the dispersion. In general, sonication is superior to shear mixing, especially for low-viscosity systems [33], where conventional mixing does not create high enough strain rates to disintegrate the CNT aggregates.

Another issue in the manufacturing of films using NFC is the shrinkage and distortion of the structure because of faster evaporation rate on surface than the mass transport of moisture within the material. When strong enough gradient occurs, film distortions emerge because of local stresses [34,35].

One widely used method for CNT dispersion is the non-covalent method. In this method, chemical moieties are adsorbed onto the surface of CNTs, the CNTs are non-covalently dispersed in a water medium, and the resultant mixture is sonicated in the presence of the moieties, namely surfactants. Surfactants are a group of organic compounds that have a hydrophilic head and a hydrophobic tail, and they are commonly used as detergents, wetting agents, emulsifiers, foaming agents and dispersants. The advantage of the non-covalent method lies in the fact that it does not deteriorate the electronic structure of the CNTs' graphitic shells, maintaining their high electrical conductivity. Good dispersion can be achieved by having a mixture of both nanocellulose and carbon nanotubes with the help of surfactants, as the surfactants lower the interfacial free energy between the particles. Table 1 lists information about surfactants and their properties used in this study.

Table 1. Surfactants used in this study.

\begin{tabular}{|c|c|c|c|}
\hline Product Name & Triton $^{\mathrm{TM}} \mathrm{x}-100$ & Pluronic $^{\circledR}$ F-127 & СТАВ \\
\hline Type & Non-ionic & Non-ionic Polymeric & Cationic \\
\hline Name & Octylphenol Ethoxylate & Poloxamer & $\begin{array}{c}\text { Hexadecyltri-methylammonium } \\
\text { bromide }\end{array}$ \\
\hline Chemical Structur & & 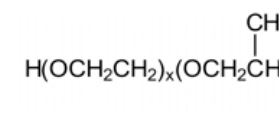 & $\underset{\mathrm{CH}_{3}^{\mathrm{N}^{+}}}{\mathrm{CH}_{3}} \mathrm{CH}_{3}^{\mathrm{CHBr}^{-}}$ \\
\hline $\begin{array}{l}\text { Critical Micelle } \\
\text { Concentration }\end{array}$ & $\begin{array}{c}0.2-0.9 \mathrm{mM} \\
(20-25)^{\circ} \mathrm{C} \\
\end{array}$ & $\begin{array}{l}950-1000 \mathrm{ppm} \\
\left(25^{\circ} \mathrm{C}\right)\end{array}$ & $\begin{array}{c}0.92 \mathrm{mM} \\
(20-25){ }^{\circ} \mathrm{C}\end{array}$ \\
\hline HLB value & 13.5 & 22 & 10 \\
\hline
\end{tabular}

In the current literature, there are several different types of surfactants used for dispersing nanocellulose and carbon nanotubes. Choosing a surfactant type for effective dispersion of nanotubes through surfactant adsorption is complicated, as the results in the published literature often give contradictory results. For example, some researchers [29] have suggested that ionic surfactants are preferable for creating aqueous dispersions. However, the non-ionic surfactant Triton X-100 was shown to be a better surfactant than the anionic surfactant SDS, which was attributed to the $\pi-\pi$ stacking ability of the former. The quality of the NFC-CNT dispersion is dependent on the nature of the surfactant, the concentration and the type of interactions between the surfactant and dispersing particles [36]. It has been stated that, for dispersing CNTs it is preferable for the surfactant to have a relatively high HLB (hydrophilic-lipophilic balance) value [29]. This assumption was proven false in our previous study [37]. Not only are the surfactant's nature and energy carried into the dispersed system, but the concentration of the surfactant also has a crucial role in the dispersion process [38]. Too high a surfactant concentration may negatively affect conductivity properties by blocking off the charge transport through the CNT network [39]. In addition, a low surfactant concentration can cause re-aggregation, because a sufficient amount is required to cover CNT surfaces to prevent re-aggregation $[39,40]$. It has been shown that an efficient CNT dispersion is only possible when the surfactant concentration is above the critical micelle concentration (CMC) value [41-44]. In some cases, the surfactant concentration is reported to be higher than the (CMC), but no micelle structures are 
observed in the dispersion. Presumably, most of the surfactant has been adsorbed onto the surface of the CNTs [40]. In other cases, surfactants can prefer surfactant-surfactant interactions over spreading on the CNT surface [45]. It has also been reported that dispersing agents can form stable dispersions below and equal to their CMC limit [46-49]. Moreover, it has been noted that commonly, the best results can be reached with a concentration of $0.5 \mathrm{CMC}$ and that any further increase in the concentration of surfactant has only a minor effect [48].

The ISO 14887:2000(E) standard can be used to determine prospective dispersing agents for both cellulose and carbon. We can categorize nanocellulose and CNTs as solids. In that case, when using water as liquid, the category of suitable dispersing agent would be a poly ethylene-oxide (PEO)/alcohol for $\mathrm{CNT}$ and $\mathrm{PEO} /$ poly propylene oxide (PPO) copolymer for nanocellulose. The standard also provides information about commercial surfactants that fall into the mentioned categories. PEO/PPO copolymer is a suitable surfactant for nanocellulose. The standard denotes that a commercial equivalent is Pluronic ${ }^{\circledR}$. In the case of CNTs, one example of alkyl phenoxy PEO ethanol dispersing agent is Triton ${ }^{\mathrm{TM}}$.

The typical approach to the manufacturing of conductive cellulose-CNT films has been to increase CNT weight percentage without optimizing the dispersion procedure or the used surfactants. Also, the effect of the particular ratios of the cellulose, CNT and surfactant toward each other has not been fully investigated. Even though ultrasonication is widely used for the dispersion and stabilization of CNTs, there is not a standard procedure for the sonication process, and different research groups have applied different sonication treatments to their samples. Sonication can cause chemical functionalization but it can also cause defects and breakage of CNTs [1,50-52]. This will further affect the performance of CNT-based materials and their applications. It has been found in the current literature that sonication parameters such as sonicator type, sonication time and temperature control vary significantly, with reported sonication times ranging from $2 \mathrm{~min}$ with tip sonication to $20 \mathrm{~h}$ for bath sonication. Dassios et al. [53] attempted to optimize the sonication parameters for the dispersion of MWCNTs in an aqueous solution. Two critical questions concerning the homogeneity of aqueous suspensions of carbon nanotubes by ultrasonic processing were identified; namely, the dependence of dispersion quality on the duration and intensity of sonication and the identification of the appropriate conditions for retaining the highly desirable initial aspect ratio of the free-standing tubes in the dispersed state. Fuge et al. [54] studied the effect of different ultrasonication parameters (time, amplitude) on undoped and nitrogen-doped MWCNTs in aqueous dispersions and found a nearly linear decrease of the arithmetic mean average in MWCNT length with increasing ultrasonication time.

The aim of this study was to optimize the conductivity of NFC-CNT nanocomposite films using a minimum amount of processing steps (e.g., without centrifugal processing of dispersion or pressing of the film), materials and chemicals. In this paper, NFC and multiwall carbon nanotubes (MWCNT) were used to prepare composite films and study the effect of the sonication energy and surfactant type on the electrical conductivity of the nanocomposite. In addition, we investigated the removal of the surfactant from the nanocomposites and the subsequent effect on the electrical conductivity. To our knowledge this is a novel approach and has not been reported previously. The conductivity properties of the nanocomposites were studied as a function of the used sonication energy amount, as well as with and without the presence of surfactant.

\section{Results}

The impact of sonication energy on electrical conductivity was one of the processing parameters with the highest interest in this study. This was due to the lack of previous research in the current literature. Also, our results show that the surfactant type and sonication energy play a major role in achieving excellent conductivity. In addition to the previously mentioned parameters, removal of surfactant can enhance conductivity values toward levels never seen or reported.

Overall, the shelf-life of the sonicated dispersion samples was significantly long, since samples remained unchanged before the film preparation. Also, sedimentation was not detected, based on the fact that conductivity values were at the same level when measured from both sides of the 
nanocomposite films. The appearance of the sonicated dispersion samples was identical; however, the consistency and visually inspected viscosity varied with increasing sonication energy. This was observed with Triton X-100 and Pluronic F-127 samples but not in cetyl trimethylammonium bromide (CTAB) surfactant-containing dispersions.

\subsection{Conductivity of NFC-CNT Nanocomposite Films}

Electrical conductivity of the even and uniform centrifugally cast films was measured using the four-probe measuring technique. With this method, it is possible to minimize the contact resistances and thus provide more accurate conductivity measurements than for the commonly used two-terminal measurement. The sheet resistances of prepared and cut nanocomposite films (size $30 \mathrm{~mm} \times 30 \mathrm{~mm}$ ) were measured using a four-point probe setup made in-house and a multimeter (Keithley 2002, Tektronix, Inc., Beaverton, OR, USA) in four-wire mode. The probes were placed in line, with equal $3 \mathrm{~mm}$ spacing. The four-probe setup is described elsewhere in detail [55]. The conductivity measurements were carried out using a $1 \mathrm{~mA}$ current and voltage was measured. Measurements were taken before and after removal of surfactant.

The selection of the most functional surfactant was an important aspect in this study. This selection was determined based on the sheet resistance measurements. The effect of different surfactants and sonication energy on conductivity is shown in Figure 1. From the conductivity diagrams, the effect of surfactant type can be visually observed and estimated.

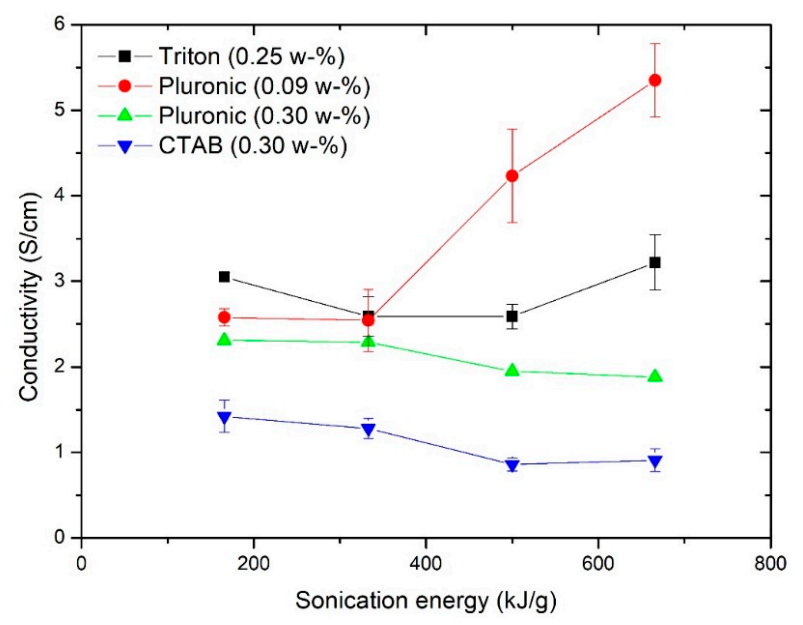

Figure 1. Conductivity of nanocomposite films processed using surfactants Triton X-100, Pluronic F-127 and cetyl trimethylammonium bromide. Concentration in weight percentages.

According to the standard, our assumption was that non-ionic surfactants would be the most promising surfactants. This was clearly the case, since the films made with surfactants Triton X-100 and Pluronic F-127 outperformed the films made with ionic surfactant CTAB.

Visual observations made with CTAB aqueous dispersion samples after sonication indicated that these samples did not gelate even with a higher amount of sonication energy per dry mass $(666 \mathrm{~kJ} / \mathrm{g})$. This suggests that the dispersion process may not have been entirely successful, since samples had different consistencies and visually separate particles. The ionic surfactant (CTAB) was used to manufacture films at a 1 to 1 ratio of dry mass content of NFC and CNT. The conductivity of films processed using $\mathrm{CTAB}$ decreased as the sonication increased from almost $1.5 \mathrm{~S} / \mathrm{cm}$ to less than $0.90 \mathrm{~S} / \mathrm{cm}$. The conductivity diagram of these films was different in its nature; the highest values were measured with the lowest amount of sonication energy.

Based on the standard Pluronic F-127, surfactant should be compatible with cellulosic materials. The first set of Pluronic F-127 nanocomposite films were done with a 1 to 1 ratio to dry mass content (0.30 wt. \%). Results show that conductivity is decreasing as a function of sonication energy. Based on 
this finding, another set of films was manufactured using a surfactant concentration below the CMC limit $(0.09 \mathrm{wt}$. \%). Conductivity results for this set of samples show higher conductivity values than films manufactured using a surfactant concentration higher than the CMC value $(0.30 \mathrm{wt}$. \%). Using Pluronic F-127 surfactant, the highest conductivity for nanocomposite films was achieved using a sonication energy of $666 \mathrm{~kJ} / \mathrm{g}$. When comparing values of films below and above CMC value the difference is sensational $5.36 \mathrm{~S} / \mathrm{cm}(0.09 \mathrm{wt}$. \%) versus $1.88 \mathrm{~S} / \mathrm{cm}(0.30 \mathrm{wt} . \%)$.

For Triton, the highest conductivity value of $3.37 \mathrm{~S} / \mathrm{cm}$ was achieved with $666 \mathrm{~kJ} / \mathrm{g}$ sonication energy. It should be noted that almost the same conductivity result $(3.02 \mathrm{~S} / \mathrm{cm})$ was achieved using just $166 \mathrm{~kJ} / \mathrm{g}$ of sonication energy.

\subsection{Effect of Surfactant Removal}

Conductivity measurements were also carried out after the removal of the surfactant used in the dispersing phase. Triton X-100 and Pluronic F-127 films were acetone treated and CTAB films were treated with ethanol. It can be clearly seen in Figure 2 that removal of surfactant has a strong effect. Removal of surfactant from films made with CTAB increased the conductivity significantly; the maximum conductivity was $3.02 \mathrm{~S} / \mathrm{cm}$ for $166 \mathrm{~kJ} / \mathrm{g}$ sonication energy. However, the films expressed a decrease in conductivity at sonication energy similar to the films with the surfactants present. For Pluronic F-127 films (Figure 2c) made below the CMC limit of the surfactant, the removal of surfactant did not have a significant effect on the conductivity. Even though there was no clear trend, the film with surfactant had somewhat higher conductivity than the one where surfactant was not present. For Pluronic F-127 (0.30 wt. \%), the shape of the diagram differed from other previous sets. The films initially exhibited a decrease in conductivity as a function of increasing sonication energy, and the highest values were measured for the samples sonicated at the least energy, but also for the highest amount of sonication energy. For the samples with high conductivity, the removal of the non-ionic surfactant increased the conductivity. The highest value obtained from these measurements was $2.88 \mathrm{~S} / \mathrm{cm}$ for a sonication energy of $166 \mathrm{~kJ} / \mathrm{g}$. A dramatic increase was observed in conductivity values of films manufactured with surfactant Triton X-100 (Figure 2a): the conductivity increases from approximately $3.0 \mathrm{~S} / \mathrm{cm}$ to a value of $8.42 \mathrm{~S} / \mathrm{cm}$ when the non-ionic surfactant was removed (sample was sonicated at $666 \mathrm{~kJ} / \mathrm{g}$ ). It should be noted that the films containing the surfactant did not exhibit as strong a sensitivity to increasing sonication energy (as those without surfactant).
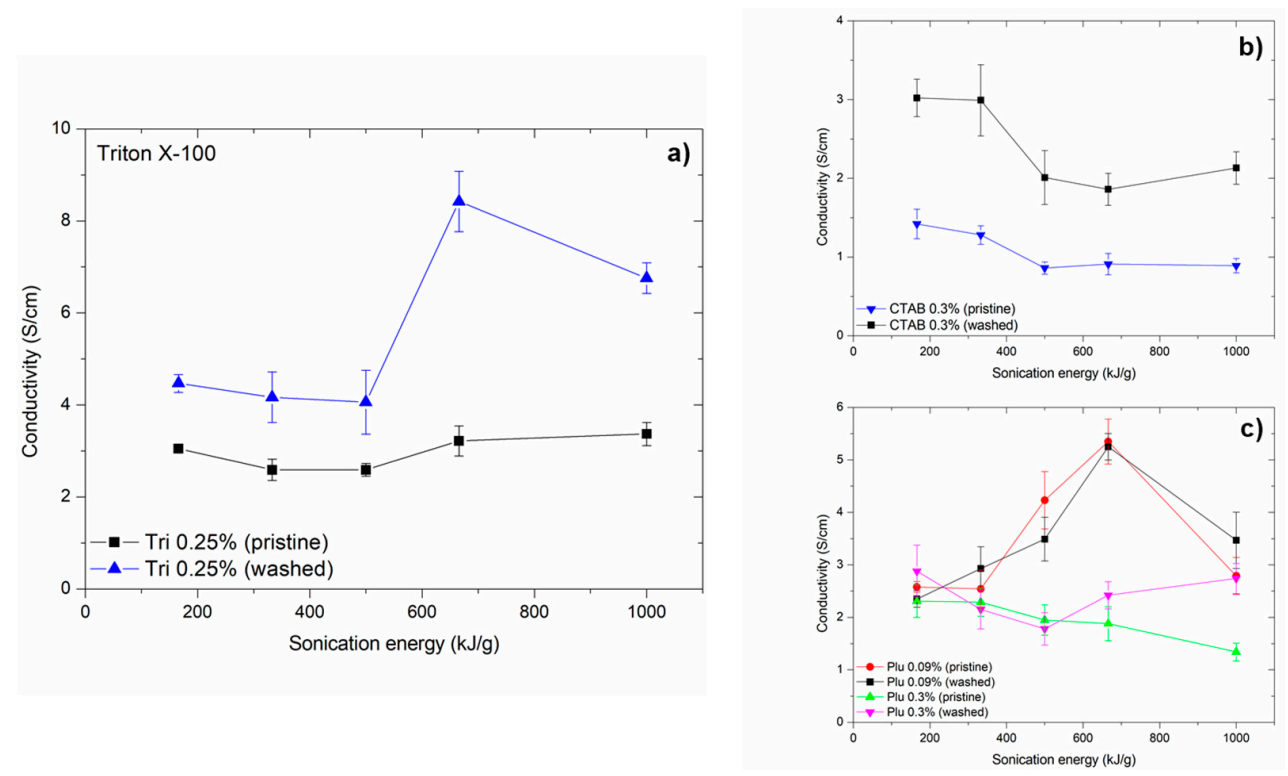

Figure 2. Conductivity of nanocomposite films before and after (a) Triton X-100 surfactant removal, (b) CTAB surfactant removal, and (c) Pluronic surfactant removal. 
It is well known that surfactants can plasticize the structure of composites and interfere with conductivity properties by situating themselves at the interface between the conductive particles and matrix. This phenomenon was demonstrated when the properties of the nanocomposite films were compared in this study. Firstly, there was a clear increase in the conductivity of the films processed using the Triton X-100 and CTAB surfactants due to the removal of the surfactant. The diagrams (pristine vs. washed) were similar in their trend, and a clear increase in terms of conductivity was observed. When using the surfactant Pluronic F-127 for processing, a clear conclusion could not be made because the conductivity diagrams did not show a corresponding, monotonic trend due to surfactant removal. However, the results showed that, when the surfactant is present in the film structure, the effect of interference by Pluronic F-127 (concentration below CMC) on the electrical conductivity is at its minimum.

\subsection{Comparison to Previous Results}

When comparing our nanocomposite film's conductivity results to previous studies, we found that our results were superior to reported values. In Figure 3 are illustrated electrical conductivity results from studies that have used native NFC and manufactured homogenous nanocomposites from it. For non-ionic surfactants, the highest conductivity value found was $0.022 \mathrm{~S} / \mathrm{cm}$ at a $10 \mathrm{wt}$. \% CNT loading [17]. In our study, the highest value was $8.42 \mathrm{~S} / \mathrm{cm}$ after removing Triton X-100 and, likewise, $5.35 \mathrm{~S} / \mathrm{cm}$ with Pluronic F-127 still present in the film.

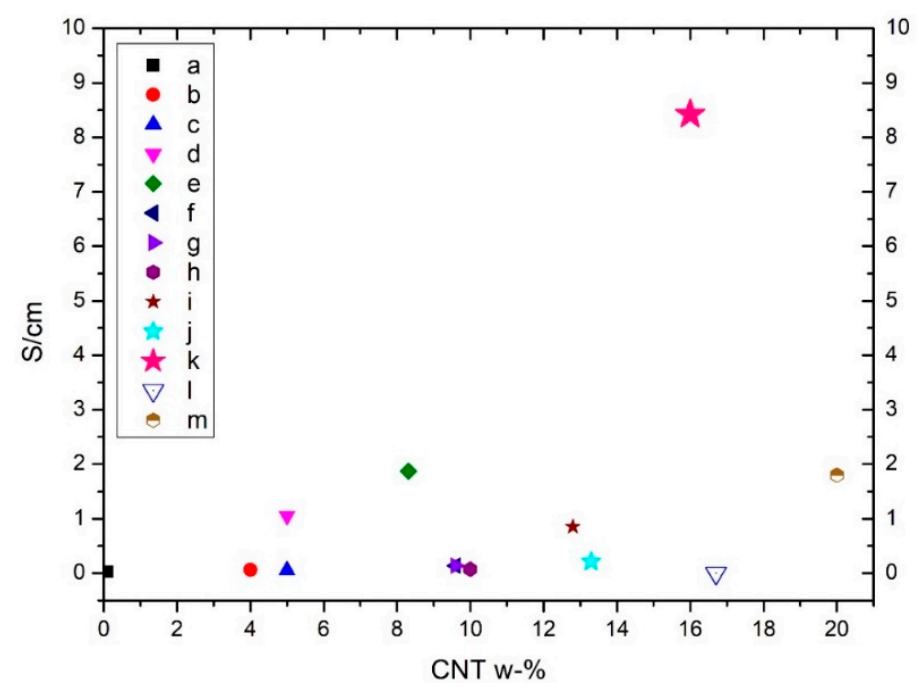

Figure 3. Comparison of obtained electrical conductivity of NFC-CNT nanocomposite films from the current literature. Pink star (letter k) refers to our data (Triton X-100), while other letters refer to a [9], b [56], c [57], d [58], e [22], f [9], g [24], h [57], i [59], j [15], 1 [32] and m [27].

Huang et al. [57] reported the results of a multiphase process which was used to accomplish a conductivity of $0.072 \mathrm{~S} / \mathrm{cm}$ using MWCNT-doping at $10 \mathrm{wt}$. $\%$ and $0.056 \mathrm{~S} / \mathrm{cm}$ with $5 \mathrm{wt} . \%$ doping with cotton linters and CTAB as a surfactant. CTAB surfactant was also used with bacterial nanocellulose and CNTs, where the conductivity was $0.027 \mathrm{~S} / \mathrm{cm}$ (MWCNT $0.1 \mathrm{wt}$. \%) [9]. Also, Yoon et al. [24] used bacterial cellulose as a matrix and obtained conductivity of $0.14 \mathrm{~S} / \mathrm{cm}$ with $9.6 \mathrm{wt}$. \% MWCNT loading. Electrical conductivity of TEMPO-oxidized cellulose films with $16.7 \mathrm{wt}$ \% concentration of MWCNTs was $0.001 \mathrm{~S} / \mathrm{cm}$, which is lower than the conductive material limit [32]. For chitosan-cellulose-CNT membranes, Xiao et al. [56] accomplished conductivity of $0.062 \mathrm{~S} / \mathrm{cm}$ with a $4 \mathrm{wt}$ \% content of MWCNTs. By using comparable materials, but by applying a filtering method, Yamakawa et al. [58] obtained a $1.05 \mathrm{~S} / \mathrm{cm}$ electrical conductivity with a $5 \mathrm{wt}$. \% MWCNT loading and 
Chen et al. $1.8 \mathrm{~S} / \mathrm{cm}$ with 20 wt. \% MWCNT. They were able to increase the conductivity to a value of $5.02 \mathrm{~S} / \mathrm{cm}$ using a chemical alkali treatment.

In addition, studies about manufacturing conductive cellulose composites via coating cellulosic filter paper with a CNT dispersion have revealed rather good results, but the consistency of the materials is not homogeneous-not exactly an integral composite. Lee et al. [15] achieved conductivity of $1.11 \mathrm{~S} / \mathrm{cm}$ using $13.3 \mathrm{wt}$. \% MWCNT. Mondal et al. [59] reported conductivity values after using a dipping method, and they reached $0.85 \mathrm{~S} / \mathrm{cm}$ with a $12.8 \mathrm{wt}$. \% carbon nanofiber (CNF) content. Fugetsu et al. [22] manufactured conductive cellulose-based composites using a traditional paper making process with 8.32 wt. \% CNT concentration and, finally, a conductivity of $1.87 \mathrm{~S} / \mathrm{cm}$ was obtained.

\subsection{Characterization of Nanocomposite Structure}

The surface and cross-section of the films processed using surfactant Triton X-100 was studied with SEM. Images were taken with Zeiss ULTRAPlus scanning electron microscope (SEM). The effect of sonication as well as the removal of surfactant were studied with the SEM images shown in Figure 4. Two samples were specifically chosen for this inspection: $166 \mathrm{~kJ} / \mathrm{g}$ and $666 \mathrm{~kJ} / \mathrm{g}$ sonication energy films containing surfactant (Figure $4 \mathrm{a}, \mathrm{c}$ ) and after removal of surfactant Triton X-100 by washing them in acetone (Figure $4 b, d$ ).
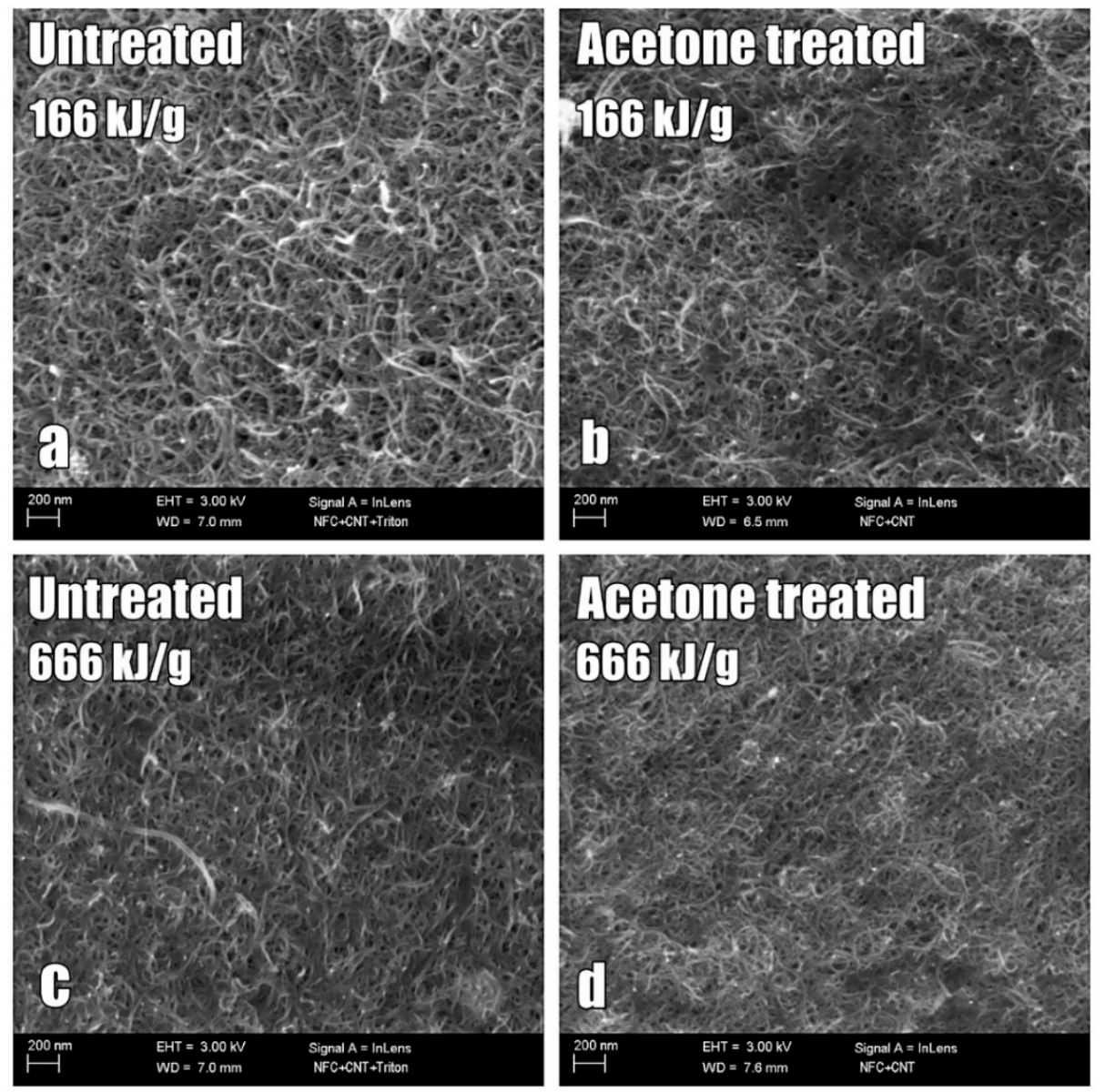

Figure 4. SEM imaging of NFC-CNT nanocomposite films surface (166 and $666 \mathrm{~kJ} / \mathrm{g}$ ) containing surfactant $(\mathbf{a}, \mathbf{c})$ and after removal of surfactant Triton $\mathrm{X}-100$ by washing them in acetone $(\mathbf{b}, \mathbf{d})$.

In the top left (Figure 4a) image, some clusters of CNTs are present in the $166 \mathrm{~kJ} / \mathrm{g}$ sonication energy sample, but not in the higher sonication energy $666 \mathrm{~kJ} / \mathrm{g}$ sample. Both films have abundant 
amount of CNTs in the surface. Here, the $166 \mathrm{~kJ} / \mathrm{g}$ film has a more porous structure than the $666 \mathrm{~kJ} / \mathrm{g}$ film. In addition, the CNTs form a more consistent network in the $666 \mathrm{~kJ} / \mathrm{g}$ film after washing the surfactant away (Figure 4 d).

The SEM images of the sonicated samples in Figure 4 showed that there were clusters present in the $166 \mathrm{~kJ} / \mathrm{g}$ sonicated film, while the higher sonicated energy sample did not have similar kinds of clusters. This indicates that, for lower sonication energies, non-dispersed particles remain in the films. This is not preferred, since the purpose is to achieve good dispersion of all the particles in the dispersion and in the films manufactured. This is an indication that the sonication process and amount of energy used affect the extent of the dispersion of particles.

The through-thickness structure of the films was also generally studied using polished cross-sections of the films embedded in epoxy. The cross-section in Figure 5 shows the CNT ends (bright contrast spots) and their even distribution in the film $(500 \mathrm{~kJ} / \mathrm{g}$ ), (Triton system) through the thickness. A slightly layered structure can be observed and concluded as a result of dispersion flow during the casting.
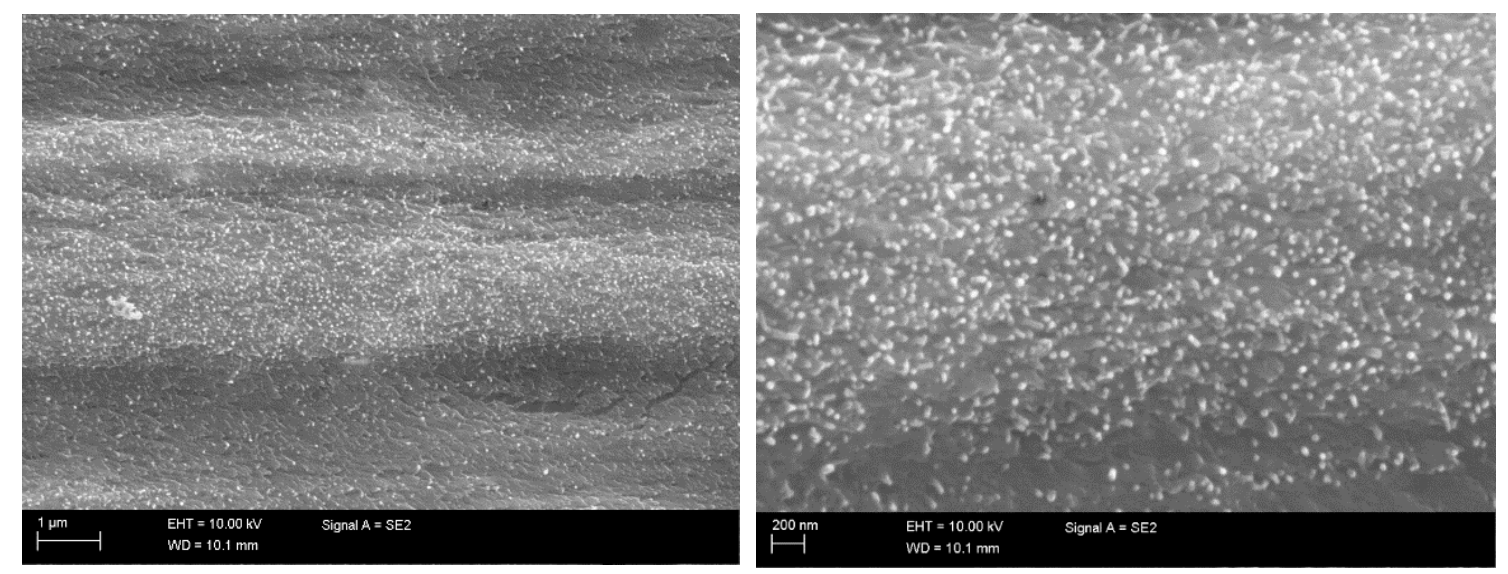

Figure 5. SEM imaging of the nanocomposite film (Triton $\mathrm{X}-100,500 \mathrm{~kJ} / \mathrm{g}$ ) cross-section when embedded in epoxy: Left side: overall structure; Right side: magnification in the center of the film.

\section{Discussion}

Ultrasonication is a widely used process to manufacture aqueous CNT dispersions. However, how much it changes the properties of dispersed particles and the medium is often overlooked. It is known that sonication can, for example, generate hydrogen peroxide from water, degrade carbon nanotubes and ultimately destroy them. Therefore, it is important that the sonication process is optimized in terms of time and power. It also needs to be noted that the dispersion process is not linear, but follows an S-curve where temporal development of dispersion quality is related to quantity of un-dispersed solid.

Due to the re-agglomeration tendency of carbon nanotubes, it is necessary to use dispersion agents, i.e., surfactants, in manufacturing aqueous dispersions. If these dispersions are later used in conductive films, it is preferable to remove the surfactant to improve CNT network formation. Although carbon nanotubes are excellent conductors, CNT networks are not. This is due their high intertubular contact resistance. The contact points act essentially as a tunneling junction for electrons that is very sensitive to distance. The efficacy of surfactants is based on acting as a spacer between tubes, so any additional distance in a conductive network is detrimental to the conductivity itself.

\section{Materials and Methods}

Three-component systems containing nanocellulose, carbon nanotubes and surfactants are used in the strong, ecologically conscious nanocomposite films of this study. The CNTs add functionality 
to the nanocellulose matrix and the surfactant enables percolation network to build and maximize conductivity properties.

In this study, the nanocellulose (NFC) production was based on mechanical disintegration of bleached hardwood kraft pulp (BHKP). First, dried commercial BHKP produced from birch was soaked in water at approximately $1.7 \mathrm{wt}$. \% concentration and dispersed using a high-shear Ystral dissolver for $10 \mathrm{~min}$ at $700 \mathrm{rpm}$. The chemical pulp suspension was predefined in a Masuko grinder (Supermasscolloider MKZA10-15J, Masuko Sangyo Co., Tokyo, Japan) at $1500 \mathrm{rpm}$ and fluidized with six passes through a Microfluidizer (Microfluidics M-7115-30, Microfluidics Corp., Newton, MA, USA) using $1800 \mathrm{MPa}$ pressure. The final material appearance of NCF was a viscous and translucent gel.

Multiwall carbon nanotubes (MWCNT, Nanocyl 7000, Nanocyl SA., Sambreville, Belgium) were purchased from Nanocyl Inc. and the product was used in the state it was in when received. This type of nanotubes is produced via catalytic chemical vapor deposition (CCVD). Concentration of CNTs was kept constant at $16 \mathrm{wt}$. \% in the nanocomposite films, so the effects of sonication energy and surfactant type to the conductivity properties are more visible.

Three surfactants were chosen based on their ionic nature and standard: non-ionic Triton X-100 and Pluronic F-127, and anionic cetyl triammonium bromide (CTAB). Surfactants were purchased from Sigma-Aldrich (Merck KGaA, Darmstadt, Germany). The surfactants were diluted in deionized water to form solutions with variating dissolutions $(1,2.5,10 \mathrm{wt}$. \%).

\section{Preparation of NFC-CNT Aqueous Dispersion}

The NFC and CNT were sonicated simultaneously and after sonication no centrifuge was used so that the preparation of aqueous dispersions could be achieved using a minimum amount of processing phases. NFC-CNT aqueous dispersions with a total volume of $80 \mathrm{~mL}$ were prepared. One set contained NFC (0.25 wt. \%), CNTs $(0.05 \mathrm{wt}$. \%), deionized water and one of the selected surfactants (Triton X-100, 0.25 wt. \%, Pluronic F-127, 0.09 wt. \% and 0.3 wt. \% and CTAB 0.30 wt. \%). Details about preparation produce are showed as Figure 6.

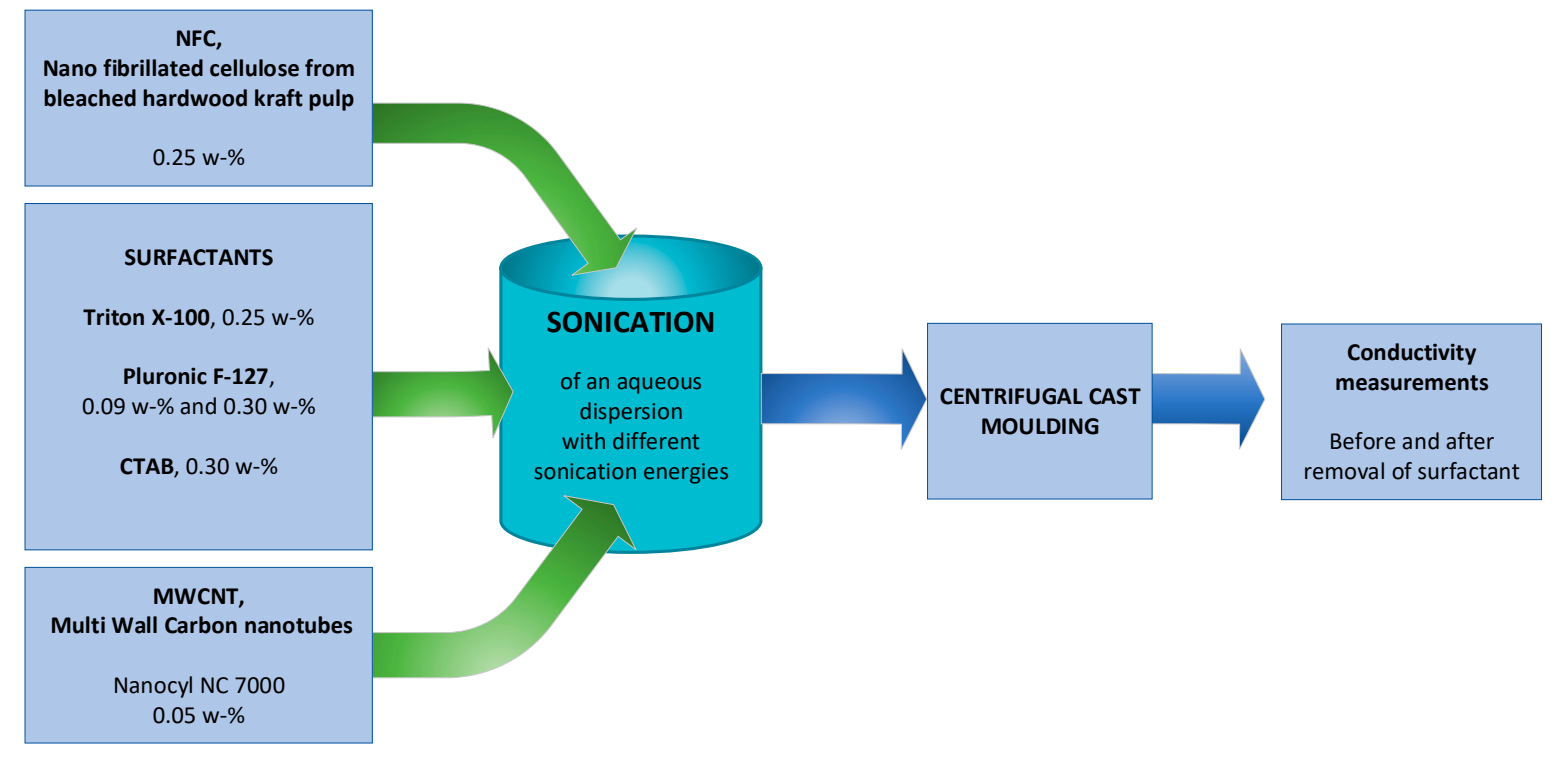

Figure 6. Preparation procedure of NFC-CNT dispersion and nanocomposite films.

The total dry mass for all the dispersions was $0.24 \mathrm{~g}$. The sonication of the dispersion samples was performed with a tip horn (ø $12.7 \mathrm{~mm}$ ) sonicator Q700 (QSonica LLC., Newton, CT, USA) in $100 \mathrm{~mL}$ glass beakers. The sonication amplitude of vibration (50\%) was kept constant. The power output remained between 60 and $70 \mathrm{~W}$ for every sonication. The system included a water bath to keep 
samples cool during the sonication so that temperature would not rise above $30^{\circ} \mathrm{C}$. The water bath was cooled by circulating cooling glycerol through a chiller (PerkinElmer C6 Chiller, PerkinElmer Inc., Waltham, MA, USA). Samples were sonicated for four different amounts of energies per dry mass, respectively 166, 333, 500 and $666 \mathrm{~kJ} / \mathrm{g}$, which corresponded to energies of 40, 80, 120 and $160 \mathrm{~kJ}$. Unsonicated samples manufactured using all three surfactants were not homogenous, and this is why film formation was unsuccessful and not analyzed.

\section{Conclusions}

The typical approach to the manufacturing of conductive cellulose-CNT films has been to increase CNT weight percentage without optimizing the dispersion procedure. In this study, NFC and multiwall carbon nanotubes (MWCNT) were used to prepare composite films using a minimum number of processing phases (e.g., no centrifugal dispersion or pressing of the film were used), materials and chemicals. The amount of CNTs was $0.05 \mathrm{wt} . \%$ in dispersion and $16 \mathrm{wt} . \%$ in the film after the evaporation of water in ratio to dry mass content of NFC and CNT. The effect of surfactant type (Triton X-100, Pluronic F-127 and CTAB) and sonication energy on the electrical conductivity of NFC-CNT nanocomposite films was investigated to identify optimal processing conditions for high conductivity of the nanocomposite. A conductivity of $5.36 \mathrm{~S} / \mathrm{cm}$ was achieved by using Pluronic F-127 surfactant and $666 \mathrm{~kJ} / \mathrm{g}$ of sonication energy. In addition, removal of the surfactant from film and its effect on the electrical conductivity was studied. A dramatic increase in conductivity values from approximately $3.0 \mathrm{~S} / \mathrm{cm}$ to a value of $8.42 \mathrm{~S} / \mathrm{cm}$ was observed for films manufactured with surfactant Triton X-100. Conductivity diagrams of the nanocomposite films show that sonication affects the electrical performance of the films. SEM images of sonicated samples showed that the films sonicated at $166 \mathrm{~kJ} / \mathrm{g}$ have a more porous structure than the films sonicated at higher energy. The imaging also showed that the CNTs form a more consistent network with a combination of high sonication energy and surfactant removal. It can be concluded that the following parameters significantly affect the conductivity of NFC-CNT nanocomposite films:

(a) Surfactant type

(b) Surfactant concentration

(c) Sonication energy

(d) Removal of the used surfactant

(e) Film processing technique

To summarize, we manufactured nanocomposite films with exemplary conductivity in comparison to reported research and this was achieved by optimizing processing parameters and materials. Further research on the surfactant types and concentration can lead to better dispersion of the CNTs and therefore even higher conductivity.

Author Contributions: S.S. and P.K. conceived and designed the experiments; S.S. and A.R. performed the experiments and analyzed the data; V.K. and A.H. contributed materials; S.S., A.R., P.K., S.T. and K.R.R. wrote the paper. M.K. and J.V. coordinated the project aims in accordance to publication specific actions and delegation.

Funding: This research received no external funding.

Acknowledgments: This work was funded by Tekes (Finnish Funding Agency for Innovation) through a strategic opening entitled Design Driven Value Chains in the World of Cellulose (DWoC 2.0). We acknowledge the contributions of Jarmo Laakso and Essi Sarlin for SEM imaging.

Conflicts of Interest: The authors declare no conflict of interest.

\section{References}

1. Huang, J.C. Carbon black filled conducting polymers and polymer blends. Adv. Polym. Technol. 2002, 21, 299-313. [CrossRef]

2. Ma, P.C.; Siddiqui, N.A.; Marom, G.; Kim, J.K. Dispersion and functionalization of carbon nanotubes for polymer-based nanocomposites: A review. Compos. Part A Appl. Sci. Manuf. 2010, 41, 1345-1367. [CrossRef] 
3. Pereira, C.; Nóvoa, P.J.R.O.; Calard, V.; Forero, S.; Hepp, F.; Pambaguian, L. Characterization of Carbon Nanotube Papers Infused with Cyanate-Ester Resin. In Proceedings of the ICCM International Conference on Composite Materials, Edinburgh, UK, 27-31 July 2009.

4. Layek, R.K.; Das, A.K.; Park, M.J.; Kim, N.H.; Lee, J.H. Enhancement of physical, mechanical, and gas barrier properties in noncovalently functionalized graphene oxide/poly(vinylidene fluoride) composites. Carbon N. Y. 2015, 81, 329-338. [CrossRef]

5. Palola, S.; Sarlin, E.; Kolahgar Azari, S.; Koutsos, V.; Vuorinen, J. Microwave induced hierarchical nanostructures on aramid fibers and their influence on adhesion properties in a rubber matrix. Appl. Surf. Sci. 2017, 410, 145-153. [CrossRef]

6. Hamedi, M.M.; Hajian, A.; Fall, A.B.; Hkansson, K.; Salajkova, M.; Lundell, F.; Wgberg, L.; Berglund, L.A. Highly conducting, strong nanocomposites based on nanocellulose-assisted aqueous dispersions of single-wall carbon nanotubes. ACS Nano 2014, 8, 2467-2476. [CrossRef] [PubMed]

7. Tuukkanen, S.; Streiff, S.; Chenevier, P.; Pinault, M.; Jeong, H.J.; Enouz-Vedrenne, S.; Cojocaru, C.S.; Pribat, D.; Bourgoin, J.P. Toward full carbon interconnects: High conductivity of individual carbon nanotube to carbon nanotube regrowth junctions. Appl. Phys. Lett. 2009, 95, 113108. [CrossRef]

8. Haghi, A.K.; Thomas, S. Carbon Nanotubes: Theoretical Concepts and Research Strategies for Engineers; Apple Academic Press: Waretown, NJ, USA, 2015.

9. Jung, R.; Kim, H.-S.; Kim, Y.; Kwon, S.-M.; Lee, H.S.; Jin, H.-J. Electrically Conductive Transparent Papers Using Multiwalled Carbon Nanotubes. J. Polym. Sci. Part B Polym. Phys. 2008, 46, 1235-1242. [CrossRef]

10. Haghi, A.K.; Zaikov, G.E. Advanced Nanotube and Nanofiber Materials; Nova Science Publishers, Inc.: New York, NY, USA, 2012; ISBN 978-1-62-081201-3.

11. Hoeng, F.; Denneulin, A.; Bras, J. Use of nanocellulose in printed electronics: A review. Nanoscale 2016, 8, 13131-13154. [CrossRef] [PubMed]

12. Gardner, D.J.; Oporto, G.S.; Mills, R.; Samir, M.A.S.A. Adhesion and surface issues in cellulose and nanocellulose. J. Adhes. Sci. Technol. 2008, 22, 545-567. [CrossRef]

13. Kuzmenko, V.; Naboka, O.; Haque, M.; Staaf, H.; Göransson, G.; Gatenholm, P.; Enoksson, P. Sustainable carbon nanofibers/nanotubes composites from cellulose as electrodes for supercapacitors. Energy 2015, 90, 1490-1496. [CrossRef]

14. Lehtimäki, S.; Tuukkanen, S.; Pörhönen, J.; Moilanen, P.; Virtanen, J.; Honkanen, M.; Lupo, D. Low-cost, solution processable carbon nanotube supercapacitors and their characterization. Appl. Phys. A Mater. Sci. Process. 2014, 117, 1329-1334. [CrossRef]

15. Lee, T.W.; Lee, S.E.; Jeong, Y.G. Carbon nanotube/cellulose papers with high performance in electric heating and electromagnetic interference shielding. Compos. Sci. Technol. 2016, 131, 77-87. [CrossRef]

16. Yun, S.; Kim, J. Multi-walled carbon nanotubes-cellulose paper for a chemical vapor sensor. Sens. Actuators B Chem. 2010, 150, 308-313. [CrossRef]

17. Qi, H.; Liu, J.; Pionteck, J.; Pötschke, P.; Mäder, E. Carbon nanotube-cellulose composite aerogels for vapour sensing. Sens. Actuators B Chem. 2015, 213, 20-26. [CrossRef]

18. Qi, H.; Mäder, E.; Liu, J. Unique water sensors based on carbon nanotube-cellulose composites. Sens. Actuators B Chem. 2013, 185, 225-230. [CrossRef]

19. Wang, M.; Anoshkin, I.V.; Nasibulin, A.G.; Korhonen, J.T.; Seitsonen, J.; Pere, J.; Kauppinen, E.I.; Ras, R.H.A.; Ikkala, O. Modifying native nanocellulose aerogels with carbon nanotubes for mechanoresponsive conductivity and pressure sensing. Adv. Mater. 2013, 25, 2428-2432. [CrossRef] [PubMed]

20. Oya, T.; Ogino, T. Production of electrically conductive paper by adding carbon nanotubes. Carbon N. Y. 2008, 46, 169-171. [CrossRef]

21. Hu, L.; Choi, J.W.; Yang, Y.; Jeong, S.; La Mantia, F.; Cui, L.-F.; Cui, Y. Highly conductive paper for energy-storage devices. Proc. Natl. Acad. Sci. USA 2009, 106, 21490-21494. [CrossRef] [PubMed]

22. Fugetsu, B.; Sano, E.; Sunada, M.; Sambongi, Y.; Shibuya, T.; Wang, X.; Hiraki, T. Electrical conductivity and electromagnetic interference shielding efficiency of carbon nanotube/cellulose composite paper. Carbon N. Y. 2008, 46, 1256-1258. [CrossRef]

23. Imai, M.; Akiyama, K.; Tanaka, T.; Sano, E. Highly strong and conductive carbon nanotube/cellulose composite paper. Compos. Sci. Technol. 2010, 70, 1564-1570. [CrossRef]

24. Yoon, S.H.; Jin, H.J.; Kook, M.C.; Pyun, Y.R. Electrically conductive bacterial cellulose by incorporation of carbon nanotubes. Biomacromolecules 2006, 7, 1280-1284. [CrossRef] [PubMed] 
25. Toomadj, F.; Farjana, S.; Sanz-Velasco, A.; Naboka, O.; Lundgren, P.; Rodriguez, K.; Toriz, G.; Gatenholm, P.; Enoksson, P. Strain sensitivity of carbon nanotubes modified cellulose. Procedia Eng. 2011, 25, 1353-1356. [CrossRef]

26. Liu, Y.; Liu, D.; Ma, Y.; Sui, G. Characterization and properties of transparent cellulose nanowhiskers-based graphene nanoplatelets/multi-walled carbon nanotubes films. Compos. Part A Appl. Sci. Manuf. 2016, 86, 77-86. [CrossRef]

27. Chen, C.; Mo, M.; Chen, W.; Pan, M.; Xu, Z.; Wang, H.; Li, D. Highly conductive nanocomposites based on cellulose nanofiber networks via $\mathrm{NaOH}$ treatments. Compos. Sci. Technol. 2018, 156, 103-108. [CrossRef]

28. Hilding, J.; Grulke, E.; George Zhang, Z.; Lockwood, F. Dispersion of Carbon Nanotubes in Liquids. J. Dispers. Sci. Technol. 2003, 24, 1-41. [CrossRef]

29. Vaisman, L.; Wagner, H.D.; Marom, G. The role of surfactants in dispersion of carbon nanotubes. Adv. Colloid Interface Sci. 2006, 128-130, 37-46. [CrossRef] [PubMed]

30. Rastogi, R.; Kaushal, R.; Tripathi, S.K.; Sharma, A.L.; Kaur, I.; Bharadwaj, L.M. Comparative study of carbon nanotube dispersion using surfactants. J. Colloid Interface Sci. 2008, 328, 421-428. [CrossRef] [PubMed]

31. Bai, J.B.; Allaoui, A. Effect of the length and the aggregate size of MWNTs on the improvement efficiency of the mechanical and electrical properties of nanocomposites-Experimental investigation. Compos. Part A Appl. Sci. Manuf. 2003, 34, 689-694. [CrossRef]

32. Salajkova, M.; Valentini, L.; Zhou, Q.; Berglund, L.A. Tough nanopaper structures based on cellulose nanofibers and carbon nanotubes. Compos. Sci. Technol. 2013, 87, 103-110. [CrossRef]

33. Huang, Y.Y.; Terentjev, E.M. Dispersion and rheology of carbon nanotubes in polymers. Int. J. Mater. Form. 2008, 1, 63-74. [CrossRef]

34. Gimåker, M.; Östlund, M.; Östlund, S.; Wågberg, L. Influence of beating and chemical additives on residual stresses in paper. Nord. Pulp Pap. Res. J. 2011, 26, 445-451. [CrossRef]

35. Baez, C.; Considine, J.; Rowlands, R. Influence of drying restraint on physical and mechanical properties of nanofibrillated cellulose films. Cellulose 2014, 21, 347-356. [CrossRef]

36. Rosen, M.J. Surfactants and Interfacial Phenomena; John Wiley \& Sons, Inc.: Hoboken, NJ, USA, 2004; ISBN 978-0-47-147818-8.

37. Keinänen, P.; Siljander, S.; Koivula, M.; Sethi, J.; Vuorinen, J.; Kanerva, M. Optimized dispersion quality of aqueous carbon nanotube colloids as a function of sonochemical yield and surfactant/CNT ratio. Heliyon 2018, in press.

38. Blanch, A.J.; Lenehan, C.E.; Quinton, J.S. Optimizing Surfactant Concentrations for Dispersion of Single-Walled Carbon Nanotubes in Aqueous Solution. J. Phys. Chem. B 2010, 114, 9805-9811. [CrossRef] [PubMed]

39. Yu, J.; Grossiord, N.; Koning, C.E.; Loos, J. Controlling the dispersion of multi-wall carbon nanotubes in aqueous surfactant solution. Carbon N. Y. 2007, 45, 618-623. [CrossRef]

40. Islam, M.F.; Rojas, E.; Bergey, D.M.; Johnson, A.T.; Yodh, A.G. High weight fraction surfactant solubilization of single-wall carbon nanotubes in water. Nano Lett. 2003, 3, 269-273. [CrossRef]

41. Utsumi, S.; Kanamaru, M.; Honda, H.; Kanoh, H.; Tanaka, H.; Ohkubo, T.; Sakai, H.; Abe, M.; Kaneko, K. RBM band shift-evidenced dispersion mechanism of single-wall carbon nanotube bundles with NaDDBS. J. Colloid Interface Sci. 2007, 308, 276-284. [CrossRef] [PubMed]

42. Sun, Z.; Nicolosi, V.; Rickard, D.; Bergin, S.D.; Aherne, D.; Coleman, J.N. Quantitative Evaluation of Surfactant-stabilized Single-walled Carbon Nanotubes: Dispersion Quality and Its Correlation with Zeta Potential. J. Phys. Chem. C 2008, 112, 10692-10699. [CrossRef]

43. Maillaud, L.; Zakri, C.; Ly, I.; Pénicaud, A.; Poulin, P. Conductivity of transparent electrodes made from interacting nanotubes. Appl. Phys. Lett. 2013, 103, 263106. [CrossRef]

44. Bai, Y.; Lin, D.; Wu, F.; Wang, Z.; Xing, B. Adsorption of Triton X-series surfactants and its role in stabilizing multi-walled carbon nanotube suspensions. Chemosphere 2010, 79, 362-367. [CrossRef] [PubMed]

45. Calvaresi, M.; Dallavalle, M.; Zerbetto, F. Wrapping nanotubes with micelles, Hemimicelles, and cylindrical micelles. Small 2009, 5, 2191-2198. [CrossRef] [PubMed]

46. Geng, Y.; Liu, M.Y.; Li, J.; Shi, X.M.; Kim, J.K. Effects of surfactant treatment on mechanical and electrical properties of CNT/epoxy nanocomposites. Compos. Part A Appl. Sci. Manuf. 2008, 39, 1876-1883. [CrossRef] 
47. Bystrzejewski, M.; Huczko, A.; Lange, H.; Gemming, T.; Büchner, B.; Rümmeli, M.H. Dispersion and diameter separation of multi-wall carbon nanotubes in aqueous solutions. J. Colloid Interface Sci. 2010, 345, 138-142. [CrossRef] [PubMed]

48. Angelikopoulos, P.; Gromov, A.; Leen, A.; Nerushev, O.; Bock, H.; Campbell, E.E.B. Below the CMC. J. Phys. Chem. C 2010, 114, 2-9. [CrossRef]

49. Bonard, J.; Stora, T.; Salvetat, J.; Maier, F.; Stockli, T.; Duschl, C.; De Heer, W.A.; Forró, L.; Châtelain, A. Purification and Size-Selection of Carbon Nanotubes. Adv. Mater. 1997, 9, 827-831. [CrossRef]

50. Lu, P.; Hsieh, Y. Lo Multiwalled carbon nanotube (MWCNT) reinforced cellulose fibers by electrospinning. ACS Appl. Mater. Interfaces 2010, 2, 2413-2420. [CrossRef] [PubMed]

51. Rossell, M.D.; Kuebel, C.; Ilari, G.; Rechberger, F.; Heiligtag, F.J.; Niederberger, M.; Koziej, D.; Erni, R. Impact of sonication pretreatment on carbon nanotubes: A transmission electron microscopy study. Carbon N. Y. 2013, 61, 404-411. [CrossRef]

52. Yang, D.; Rochette, J.-F.; Sacher, E. Functionalization of Multiwalled Carbon Nanotubes by Mild Aqueous Sonication. J. Phys. Chem. B 2005, 109, 7788-7794. [CrossRef] [PubMed]

53. Dassios, K.G.; Alafogianni, P.; Antiohos, S.K.; Leptokaridis, C.; Barkoula, N.M.; Matikas, T.E. Optimization of sonication parameters for homogeneous surfactant assisted dispersion of multiwalled carbon nanotubes in aqueous solutions. J. Phys. Chem. C 2015, 119, 7506-7516. [CrossRef]

54. Fuge, R.; Liebscher, M.; Schröfl, C.; Oswald, S.; Leonhardt, A.; Büchner, B.; Mechtcherine, V. Fragmentation characteristics of undoped and nitrogen-doped multiwalled carbon nanotubes in aqueous dispersion in dependence on the ultrasonication parameters. Diam. Relat. Mater. 2016, 66, 126-134. [CrossRef]

55. Rajala, S.; Tuukkanen, S.; Halttunen, J. Characteristics of piezoelectric polymer film sensors with solution-processable graphene-based electrode materials. IEEE Sens. J. 2015, 15, 3102-3109. [CrossRef]

56. Xiao, W.; Wu, T.; Peng, J.; Bai, Y.; Li, J.; Lai, G.; Wu, Y.; Dai, L. Preparation, structure, and properties of chitosan/cellulose/multiwalled carbon nanotube composite membranes and fibers. J. Appl. Polym. Sci. 2013, 128, 1193-1199. [CrossRef]

57. Huang, H.D.; Liu, C.Y.; Zhang, L.Q.; Zhong, G.J.; Li, Z.M. Simultaneous reinforcement and toughening of carbon nanotube/cellulose conductive nanocomposite films by interfacial hydrogen bonding. ACS Sustain. Chem. Eng. 2015, 3, 317-324. [CrossRef]

58. Yamakawa, A.; Suzuki, S.; Oku, T.; Enomoto, K.; Ikeda, M.; Rodrigue, J.; Tateiwa, K.; Terada, Y.; Yano, H.; Kitamura, S. Nanostructure and physical properties of cellulose nanofiber-carbon nanotube composite films. Carbohydr. Polym. 2017, 171, 129-135. [CrossRef] [PubMed]

59. Mondal, S.; Ganguly, S.; Das, P.; Bhawal, P.; Das, T.K.; Nayak, L.; Khastgir, D.; Das, N.C. High-performance carbon nanofiber coated cellulose filter paper for electromagnetic interference shielding. Cellulose 2017, 24, 5117-5131. [CrossRef]

(C) 2018 by the authors. Licensee MDPI, Basel, Switzerland. This article is an open access article distributed under the terms and conditions of the Creative Commons Attribution (CC BY) license (http://creativecommons.org/licenses/by/4.0/). 